\title{
LHomme
}

LOMME Revue française d'anthropologie

$201 \mid 2012$

Varia

\section{Comptes rendus - Amériques, Asie, Maghreb, Europe}

\section{(2) OpenEdition \\ Journals}

Édition électronique

URL : http://journals.openedition.org/lhomme/23008

DOI : 10.4000//homme.23008

ISSN : 1953-8103

Éditeur

Éditions de l'EHESS

\section{Édition imprimée}

Date de publication : 23 février 2012

Pagination : 213-227

ISSN : 0439-4216

Référence électronique

"Comptes rendus - Amériques, Asie, Maghreb, Europe », L'Homme [En ligne], 201 | 2012, mis en ligne le 15 février 2014, consulté le 24 septembre 2020. URL : http://journals.openedition.org//homme/23008 ; DOI : https://doi.org/10.4000//homme.23008 


\section{A MÉ RI Q UES}

\section{Guilhem Olivier, ed.}

Símbolos de poder en Mesoamérica

México, Universidad nacional autónoma de México, 2008, 55 I p., bibl., ill., fig., cartes («Instituto de investigaciones históricas : Serie Culturas Mesoamericanas » 5).

C

LIVRE RASSEMBLE les contributions présentées dans le symposium "Image, symboles et métaphores du pouvoir en Mésoamérique ", lors du $51^{\mathrm{e}}$ Congrès international des américanistes, tenu à Santiago du Chili du 14 au 17 juillet 2003. Ses vingt-six auteurs sont des archéologues, des anthropologues, des historiens et des linguistes, et ce dialogue interdisciplinaire, encore assez rare, mérite d'être souligné. Divisé en cinq parties en fonction de la cohérence thématique des textes (notions, représentations, rituels, hommes de pouvoir, ainsi que pouvoir et résistance en Mésoamérique), l'ouvrage est forcément inégal. Une série d'articles ne fait pas nécessairement un livre et on peut regretter l'absence d'une conclusion de synthèse qui aurait été bien utile aux non-spécialistes de cette aire géographique et culturelle. Étant donné le foisonnement des sujets abordés, on aurait aussi aimé un index, qui permettrait une consultation plus aisée. Voilà pour ce qui est des remarques générales. Ces critiques formelles ne devraient pas, en tout cas, dissuader les lecteurs de lire ce volume dont les analyses, souvent passionnantes, ouvrent, au-delà $\mathrm{du}$ cas Mésoaméricain, des nouvelles pistes de comparaison avec d'autres cultures du continent.
Tout d'abord, il convient de rappeler brièvement les principales difficultés qui se présentent aux chercheurs désireux de se frayer un passage dans la complexité mésoaméricaine. La première est l'inextricable fusion du mythe, de l'histoire et de la propagande. Ce point n'est pas exclusif de la Mésoamérique, bien entendu, mais c'est là qu'il se pose avec le plus d'acuité. Contrairement aux autres peuples des Amériques, nous disposons, pour cette civilisation, de documents produits par les autochtones, soit des codex, soit des inscriptions peintes ou gravées, et de sources réélaborées au XVI ${ }^{\mathrm{e}}$ siècle, qui fonctionnent en quelque sorte comme des "pierres de Rosette» pour interpréter des informations obscures. L'histoire mouvementée des Mexica (ou Aztèques), un peuple nomade de la famille des Nahua, soumis aux puissants seigneurs du lac de Tezcoco avant d'en prendre leur revanche vers le milieu du $\mathrm{XV}^{\mathrm{e}}$ siècle, impliquait la nécessité pour eux de se donner une légitimité. C'est ainsi que les Mexica triomphants réécrivent l'histoire de leur errance sous la conduite infaillible de leur dieu, Huitzilipochtli, et détruisent les témoignages écrits plus anciens, dans lesquels les Aztèques n'étaient qu'un peuple de second rang. Ces distorsions se 
poursuivent à l'époque coloniale, où des chroniqueurs issus de cités opposées à leur propre vision des événements. C'est dire que les chroniques dont nous disposons ne racontent pas tout à fait la même histoire. Toute réflexion sur le pouvoir implique donc un travail critique sur les sources, qui est à l'œuvre dans plusieurs textes, notamment dans ceux de Michel Graulich et de Sylvie Peperstraete.

Une autre difficulté, qui a souvent rebuté plus d'un américaniste, réside dans le foisonnement (presque) infini de noms donnés aux dieux et à leurs attributs. Métamorphoses, redoublements, répliques, fragmentations et recompositions égarent souvent les non-spécialistes, et Huemac, Quezalcoatl et Xipe Totec, pour ne citer que trois noms qui apparaissent dans plusieurs contributions, renvoient, chacun, à des traditions différentes. En outre, les vies d'un roi, d'une cité, d'un État et d'une civilisation, sont comparables à la course du soleil, et la laideur de Topiltzin Quetzalcoatl, mais aussi du Quetzalcoatl créateur du monde alors que d'autres documents vantent sa beauté, est celle du vieillard parvenu au seuil de l'infra-monde (José Contel, p. 340).

Quels sont les noms du roi et du pouvoir qu'il exerce, ou de l'autorité qu'il incarne, dans les langues vernaculaires? Felipe Castro Gutiérrez et Cristina Monzón García (pp. 31-46) ouvrent la discussion avec un texte sur le vocabulaire des Tarasques (Michoacán). Là, le mot qui désigne l' "autorité " possède les mêmes racines que "faire peur, effrayer». Parmi d'autres associations intéressantes, citons celle qui rapproche "posséder la terre " et "apparenter" ou "se faire des parents". Marc Thouvenot (pp. 47-65) examine exhaustivement le terme de teuctli ("dignitaire» ou "seigneur»), montrant ainsi qu'il s'agit d'une qualité et non d'une charge. Les spécialistes de la région andine auront tout intérêt à lire Laura Caso Barrera et Mario Aliphat (pp. 67-84) qui analysent les vases et les libations, en termes de

symboles de pouvoir, chez les Itzaes. Il serait d'ailleurs temps de revenir à quelques fondamentaux sur l'unité des cultures amérindiennes, séparées par les excès nominalistes de ces dernières années. La première partie s'achève par deux autres textes: l'un de John et Robert Schwaller (pp. 85-94), sur la conquête et la création comme principes de pouvoir, fondé en partie sur le travail de Marshall Sahlins; l'autre de Jacques Galinier (pp. 95-107), qui reprend les bases de la théorie "psychanalytique " des Otomi en s'appuyant largement sur les notions freudiennes.

Plusieurs études abordent avec bonheur le thème de la représentation du pouvoir, en fondant leurs analyses sur une iconographie détaillée, reproduite dans le volume. Dans cette partie, les sources du $\mathrm{XVI}^{\mathrm{e}}$ siècle sont confrontées aux découvertes archéologiques effectuées dans différentes régions mésoaméricaines. La région de la Huasteca (golfe du Mexique) et ses symboles marins sont traités par Lorenzo Ochoa (pp. 133-161), qui apporte des éléments moins familiers que ceux qui apparaissent dans les livres peints des Mexica. En ce qui concerne ce dernier peuple, Eloise Quiñones Keber et AnneMarie Vié-Wohrer (respectivement, pp. 175192 et 193-229) fournissent une sorte de dictionnaire raisonné iconographique qui est très utile pour tous les chercheurs américanistes. Certes, ce corpus d'images peut ouvrir sur d'autres questions. Le pied nu du souverain, par exemple, pourrait être mis en rapport avec le déplacement, comme on le voit dans d'autres pictographies. Citons encore le texte de Michel Graulich (pp. 163-174), auteur qui a influencé la plupart des contributeurs de ce recueil, et qui donne à l'institution de la guerre sacrée son cadre historique, en analysant la réforme religieuse survenue en 1507 , marquée par un changement des dates et par l'importance prépondérante donnée au dieu Huitzilipochtli.

La troisième partie sur les rituels du pouvoir en Mésoamérique est, selon moi, la plus intéressante et la plus novatrice. 
Les rituels de clôture chez les Tarasques (Patricia Carot, pp. 231-261) remettent en question la version courante de l'histoire purépecha, fondée sur l'invasion du territoire par des nomades chichimèques. En fait, l'auteur démontre, sur la base de la documentation archéologique, que cette prétendue invasion n'est autre que le retour, plusieurs siècles plus tard et sur les lieux ancestraux, d'un peuple originaire du Michoacán obligé d'émigrer vers le nord à la suite du dessèchement du marais de Zacapu. Le texte développe également un point essentiel, celui de l'enterrement volontaire de fragments d'un site, précédant un départ. Nous trouvons très loin dans le temps et dans l'espace, dans la vallée de Caral au Pérou, au deuxième millénaire avant notre ère, une situation semblable. Les trois morts symboliques du souverain mexica traitées par Guilhem Olivier (pp. 263-291) apportent, parmi bien d'autres données, un "recouvrement", ici du roi initié par un tissu, geste équivalent à celui des paquets sacrés contenant des restes des dieux. Patrick Lesbre (pp. 293-313) reprend le thème de la chasse aux oiseaux à la sarbacane, en insistant sur les pratiques des rois de Tezcoco à l'époque coloniale. Danièle Dehouve (pp. 315-334) livre une des contributions les plus remarquables de cet ouvrage, en comparant le sacrifice du jaguar des Tlapanèques au rituel actuel du sacrifice du chat, dont la dépouille est posée sur différents niveaux d'offrandes, suivant un ordre numérique précis.

La quatrième partie porte sur des personnalités concrètes, encore que ce qualificatif se brouille quand il s'agit d'établir la réalité historique de Tlacaelel, celui qu'une certaine historiographie considère comme le destructeur des codex anciens et celui qui inaugure une nouvelle vision du monde (Sylvie Peperstraete, pp. 375-426). José Contel (pp. 337-358) approfondit le rôle complexe de Tlaloc, dieu des pluies et de la germination, mais aussi détenteur d'un pouvoir politique, fonction qui n'a pas toujours été prise en compte. Un travail de révision est encore celui de Carlos Javier González (pp. 359374) sur la figure de Xipe Totec. On trouve également dans cette partie des contributions aussi variées que la reconstruction de généalogies de chefs-gouverneurs de Mexico (María Castañeda de la Paz, pp. 393-426), ou le non-culte rendu par les indigènes aux enfants martyrs de Tlaxcala, considérés comme des traîtres par leurs compatriotes, mais vénérés par l'Église (Robert Haskett, pp. 427-440). Le livre s'achève par cinq textes assez différents, unis par la référence à la résistance, dans le sens anti-utopique classique (Federico Navarrete, pp. 475-492 et José Luis Mirafuentes Galván, pp. 493-516), deux autres sur les Indiens aujourd'hui, et un texte un peu convenu sur le couple initial sexué (en fait une divinité aux deux sexes), dans le sillage des gender studies, qui reste trop général (Stephanie Wood, pp. 517533). C'est la partie la plus décevante du livre, soit par un énoncé dépassé de la résistance indienne, soit par des généralisations sur les mouvements indiens actuels, dont l'importance aurait justifié une analyse plus fine que celle qui est apportée ici.

Ce livre, on l'aura compris, est un premier pas vers une réflexion renouvelée sur le pouvoir politique et la manipulation, mais aussi, sur l'ambiguïté de la relation sacrifiant-sacrifié, qui affleure dans plusieurs textes et qui justifierait une recherche comparative plus poussée. Les travaux à venir gagneraient à approfondir les notions utilisées, à distinguer pouvoir et autorité, qui ne sont pas des termes équivalents, ainsi que les fonctions de pouvoir exercées par les souverains et les prêtres.

Carmen Bernand 
L'

AUTEUR a déjà produit une longue liste de publications sur cette région, et l'ouvrage présenté ici réunit plusieurs idées développées antérieurement, proposant au lecteur un objet complexe tout en l'invitant à poursuivre la réflexion ainsi engagée.

Le livre s'organise en dix chapitres, auxquels s'ajoutent une introduction, une conclusion, un prologue et un épilogue, une bibliographie, un index des noms d'auteurs et un index des termes vernaculaires. Les seules illustrations sont des photographies regroupées dans un cahier central, bienvenues pour un tel sujet. On peut regretter qu'elles ne soient pas datées, car elles témoignent de terrains successifs égrenés sur une période de trente ans et pourraient livrer des indices d'analyse diachronique. De même, la présence d'un plan de ville, d'une carte et d'un schéma de déambulation des troupes aurait aidé le lecteur à situer les éléments décrits.

Le titre et le sous-titre, ainsi que les trois exergues de Rousseau, Durkheim et Barthes indiquent clairement les fils rouges de l'ouvrage: la force cohésive de la fêtespectacle, la vision collective exprimée dans le rite et le pouvoir de la théâtralité à dépasser le texte. L'auteur voit dans la fête "trois plans différents: l'expression d'une culture, le témoin d'une histoire et l'autoreprésentation d'une société» (p. 3, en italique dans le texte); il voit également une tension entre fonction spectaculaire et fonction rituelle, questionne les frontières entre théâtre et rite, et propose de «jeter les bases d'une théorie" (p. 6) à partir de l'étude d' "une grande fête urbaine annuelle observée plusieurs fois au Népal» (p. 4), la fête d'Indra à Katmandou.

L'Indra Jâtrâ est une fête néwar, groupe ethnique de plus de 1,5 million de personnes, minorité établie dans la vallée de Katmandou, représentant 5\% de la population népalaise. Cette fête synthétise hindouisme et bouddhisme du Grand Véhicule, et évoque l'Inde du Sud et l'Orissa. Elle remonte au moins au début de l'époque Malla (XIII-XIV e siècle), existe dans toutes les localités néwar de la vallée et est devenue la fête officielle népalaise. Elle requérait la présence du roi jusqu'à l'abolition de la monarchie en 2008. C'est la vieille ville, cour d'une cité unifiée par un souverain également fondateur de cette fête, qui tient lieu de décor et de scène à toute une imbrication de spectacles théâtraux, permettant d'exposer, de sortir les dieux. Tout en expliquant la difficulté d'une lecture unique de cette fête qui honore trois divinités à la fois - la déesse Kumârî, Indra et Bhairava (une forme de Shiva) -, l'auteur cherche à en dégager une unité structurelle, même incomplète, 
puis essaie de comprendre l'émotion, la perception esthétique liée - il insiste sur le fait - à l'acte de regarder.

Les formes théâtrales traditionnelles néwar dérivent du théâtre indien classique et suivent sa définition, donnée dans le traité Nâtyashâstra, d' " objet de jeu à voir et à entendre ». Pour comprendre les relations entre rite et théâtre, il est indispensable d'adopter une vision processuelle et nuancée. Gérard Toffin étend les comparaisons au monde européen contemporain, médiéval et antique : il suit Georges Bataille dans son idée de "dépense improductive " appliquée aux manifestations festives, Michel Leiris sur la possession et le théâtre, Jack Goody sur la liturgie chrétienne, Charles Malamoud sur la théâtralité du védique, Victor Turner dans son exploration du théâtre et du rituel et son concept de "drames sociaux", Clifford Geertz, enfin, pour l'usage métaphorique de la dramaturgie en domaine politique; il ajoute ses propres observations des "nouvelles fêtes médiévales" en terrain français. Finalement, il rapproche Grèce ancienne et Inde classique et contemporaine pour leurs spectacles qui doivent satisfaire les dieux et les hommes, relevant ainsi à la fois du sacré et du profane.

L'auteur démasque une tension rarement reconnue - les idées véhiculées ne reflètent qu'une idéologie dominante au sein d'une culture complexe et diversifiée - et souligne l'intérêt pour l'ethnologue de faire appel à l'indologie et à l'histoire pour affiner ses observations sociologiques. Ainsi la centralité du personnage d'"Indra, figure de la royauté » ne doit-elle pas éclipser les différences d'interprétation des rites et des mots selon les divers milieux sociaux, professionnels ou religieux. La discussion sur la pertinence d'une lecture du rite comme "champ de bataille rituel" illustre les difficultés de la mise en perspective historique et géographique (par rapport à l'Inde védique) et les risques d'anachronisme. Pourtant, l'auteur montre bien l'évolution des rituels néwar, le changement d'esprit qu'a imprimé la fête, ainsi que l'émergence d'un " ressort narratif » théâtral. La présence de rituels funéraires et l'ambiguïté de la fonction royale mise en scène posent question, d'autant que les dieux et le roi sont à la fois sujets et spectateurs, et que les deux divinités Indra et Bharaiva/Shiva sont associées.

Il s'agit bien de théâtres et de danses sacrées, dans une mosaïque de tableaux vivants, théâtre ancien classique, danses de démons et marionnettes. La procession (yâtrâ) conduit au théâtre, selon le continuum énoncé par Sylvain Lévi "procession-fête-spectacle-rite », et Gérard Toffin écarte la notion de rituel d'inversion et de dilapidation. "L'Indra Jâtrâ est une commémoration du théâtre indien primitif tel qu'il est narré dans le traité en question [Nâtyashâstra]» (p. 98). D’ailleurs, dans ce mythe d'origine du théâtre apparaît déjà l'étendard d'Indra, protecteur des acteurs et du théâtre. Qu'elles soient sculptures, peintures ou objets chargés d'un pouvoir iconographique (comme les pierres de rivière), "les "images-objets" [...] recouvrent des actes (rituels) et des mythes (narrations) » au-delà de la représentation plastique. On peut se demander si ce constat ne correspond pas au double sens du mot français "représentation"? De même, quand l'auteur rapproche Bhairava de Dionysos pour leur commune personnification des "forces sacrées de la transgression » (p. 118), ne revient-on pas à l'un des fondamentaux de la fête, pourtant écarté précédemment? Quant à la fonction du masque, on pourrait suggérer que, s'il s'applique aux dieux terribles, il s'intercale aussi entre le visage de l'acteur et la puissance divine, et terrorise encore mieux un spectateur qui ne voit plus l'acteur. Enfin, si l'on convoque Dionysos, Apollon ne devrait-il pas également être requis pour la bonne gestion des tensions et des énergies mises en jeu? Dans "Une grammaire de l'image" (pp. 124-125), Gérard Toffin présente les jalons d'une réflexion sur les principes « figuratif/aniconique, immobile/ animé " qu’il souhaiterait voir développés au sein d'une " anthropologie des images ». 
S'il s'agit bien d'un art de la religion, qui "amalgame intercession et illusion", il répond à une esthétique structurée. On pourrait ajouter qu'on y reconnaît cet effort commun et récurrent pour animer ce qui ne l'est pas, au double sens de rendre «mobile» et de donner une "âme».

Tout en suivant Louis Dumont dans sa reconnaissance de l'importance d'une organisation sociale hiérarchisée d'essence religieuse, " au cœur du système des castes hindou » (p. 128), ainsi que de la notion de pureté/impureté, l'auteur réhabilite les travaux d'Arthur Maurice Hocart et leur pertinence pour le cas des Néwar. Il reprend l'idée de Roger Caillois d'une corrélation entre fête et sacrifice, considère "la fête comme rituel sacrificiel » et y place le roi comme figure centrale. Le sous-chapitre "Le lien local » révèle un trait particulier qui mériterait peut-être d'être développé : l'appartenance à un territoire, village ou ville natale, renforce le caractère local des fêtes néwar, cette capacité d'" autonomie propre transcendant en grande partie les liens de caste et de lignée ».

Gérard Toffin dévoile finalement une analyse dynamique de cette fête (proposée dans un article précédent) à partir de la dualité de mémoires, de groupes ethniques et d'échelles temporelles qui la caractérise et qui aboutit à une véritable ambiguïté de la célébration. D’un côté, les "vaincus", Néwar de Katmandou, toutes castes confondues, attachés à Bhairava, aux processions funéraires et aux cultes locaux d'Indra, de l'autre côté, les " conquérants ", castes indo-népalaises parbatiyâ attachées au roi, à la déesse Kumâri et au mât d'Indra. En y ajoutant une dimension interethnique et même de "résistance culturelle " (p. 143), la politisation générale de la vie culturelle et les derniers développements de la nouvelle république, l'auteur aboutit à une opposition entre deux visions : révolutionnaire et maoïste, ou néwar et ethnique. La fresque historique conduit aux revendications féministes ou égalitaristes contemporaines et soutient l'observation d'une plus grande résistance à la laïcisation qu'à la patrimonialisation de la fête par le nouveau régime.

La conclusion permet à l'auteur de corroborer les travaux précurseurs de Hocart, notamment le principe "d'une origine rituelle de la plupart des institutions culturelles sociales ou même technologiques» (p. 160), l'idée que la royauté "trouve sa source dans la célébration de certains rituels d'intronisation et des sacrifices" (id.), ou encore celle de l'unité de tous les rites dans leur fonction et leur structure afin d'assurer stabilité et prospérité. En plaçant au centre de l'univers népalais la fonction royale sacrificielle, Gérard Toffin se démarque du courant structuraliste "pour qui ce n'est pas la religion qui est au fondement de la société ou de la culture, mais l'échange entre groupes sociaux et les opérations mentales " (id.). L'autre notion de base qu'il a su mettre en valeur avec brio est celle de la fonction spectaculaire : la "fête-spectacle" et l'ambiguïté de ses célébrations porteuses d'illusions et de risques. L'épilogue appelle à poursuivre l'étude de la fonction et des phénomènes spectaculaires, incitation que les anthropologues seront d'autant plus enthousiastes à suivre qu'ils ne peuvent qu'être convaincus par la richesse de cet ouvrage.

Hélène Bouvier 


\section{A G H R E B}

Baptiste Buob

La Dinanderie de Fès. Un artisanat traditionnel dans les temps modernes: une anthropologie des techniques par le film et le texte Paris, Ibis Press-Éd. de la Maison des sciences de l'homme, 2009 420 p., ill., fig., tabl., cartes, DvD encarté.

$P_{A n}$ des rapports entre tradition et artisanat, l'ouvrage nous invite à découvrir, dans une imposante monographie conduite entre 2000 et 2006, le métier de dinandier, pour lequel la "tradition authentifiée " s'accompagne d'une réduction de la technique.

L'artisan travaille à la main, dans le calme, et vend lui-même les objets qu'il fabrique. C'est à ce cliché tenace que Baptiste Buob s'oppose à partir d'une analyse détaillée de la dinanderie, menée dans la Médina de Fès. Il propose d'interroger les valeurs morales et les savoirfaire des dinandiers unis par un réseau d'entraide, alternative "aux affres de la modernité ", mais aussi "frein au développement économique» (p. 27). L'ouvrage mêle fort subtilement ethnographie filmique (un DvD joint au texte comprend 4 films) et analyse anthropologique, deux supports autonomes mais complémentaires.

La première partie, à caractère historique, traite des influences et des mutations que connaît la dinanderie. Baptiste Buob décrit l'institution de la his $b a$ - structure à la base de l'organisation des métiers - et sa réforme qui a supprimé la cohérence des métiers. Les commerces, eux, se développent, c'est le signe des formes nouvelles du capitalisme. Si, à Fès, l'artisanat est en déclin du fait de la déstructuration des bases organisationnelles, un nouveau type d'acteur, fort bien dépeint par l'auteur, l' "homme à la sacoche", le détenteur de l'argent, va s'imposer au maître du savoir. Un nouveau rapport d'exploitation lie ouvriers et élite patronale.

C'est l'introduction du thé au Maroc (au $\mathrm{XVII}{ }^{\mathrm{e}}$ siècle) et sa généralisation progressive (au XIX siècle) qui ont relancé la dinanderie via la production d'objets nécessaires à sa fabrication et à son service. Dans les années 1970 , la prise de conscience de la désagrégation du patrimoine marocain et de l'artisanat fassi a conduit la politique publique marocaine, initiée pendant le Protectorat, à promouvoir les produits touristiques. Cependant, "la mécanisation ne répond pas à une logique d'industrialisation de l'ensemble de l'appareil productif de la dinanderie» (p. 99).

L'expression «l'artisan est torchon ou lampion " exprime le rôle paradoxal qu'on lui donne, entre dégradation et valorisation. Les représentations stéréotypées de la Médina et de la dinanderie sont chaque fois relevées, telles qu'encore "abri conservateur pour les savoirs ancestraux» (p. 101). Or, le thé n'est pas un produit si ancien contrairement à ce que laissent penser les discours de certains Marocains. C'est ainsi que «la dinanderie n'est pas une tradition ancestrale, elle est devenue traditionnelle» (p. 102). 
La deuxième partie traite du travail et des objets relevant de cette forme d'industrialisation hybride, faite de mécanisation mais utilisant aussi une abondante maind'œuvre, et dont la dinanderie - devenue une des activités principales de la Médinaest une belle illustration. Baptiste Buob relève la distinction entre les objets en laiton jaune, qui plaisent aux Marocains et ceux en cuivre rouge que préferent les touristes: "Cette opposition entre une idée occidentale et une idée locale du beau est ancienne» (p. 117). La vente indirecte dans les bazars à destination des touristes est à l'origine de prix prohibitifs, en raison $\mathrm{du}$ système de rétribution onéreux des bazaristes et des agences touristiques. Il existe aussi un fossé entre circuits de production et commercialisation.

Le monde des bazars n'a rien à voir avec celui des artisans dont les films retracent le travail. Les images peuvent être visionnées en alternance avec la lecture. Elles sont d'une grande qualité, sans commentaires, donnant au lecteur/spectateur le sentiment de côtoyer, au plus près, le monde du travail des dinandiers. L'objectif est pleinement atteint car la pénétration des sociétés au Maroc, en milieu urbain et/ou rural, dont le tourisme et l'apparente proximité donnent des images déformées, n'est pas chose aisée pour l'ethnologue. Les qualités de la production filmique et des écrits de l'auteur racontent avec évidence le temps passé, nécessairement long, si l'on veut comprendre et saisir des réalités sans cela inabordables ${ }^{1}$.

L'auteur étudie ensuite les espaces sociaux de production : atelier (pièce indépendante), fondouk ${ }^{2}$ (réunion de plusieurs ateliers) et fabrique (plusieurs phases du processus de fabrication dans des pièces contiguës). L'analyse de la disposition des postes de travail permet de comprendre la division des tâches et la spécialisation technique de la dinanderie. L'éclatement des espaces crée une grande mobilité des objets qui passent sans cesse d'un atelier à l'autre. Le lecteur est invité à une anthropologie des techniques, du travail et de l'économie, loin des représentations d'un simple « artisanat local».
Dans la troisième partie, l'auteur analyse le changement technique. Autrefois " art de battre le métal ", la dinanderie se situe entre la chaudronnerie et l'orfevrerie. La lecture et le visionnage des films s'avèrent ici très complémentaires. Le film Ovale est à ce titre édifiant. Il montre qu'il faut huit jours pour achever un plateau ovale et trente-trois dinandiers. L'évocation des différentes phases est précise : le laminage, la découpe du laiton en feuille, le traçage. L'écrit complète le film, ou l'inverse... La description des outils, décors et gestes techniques utilisés pour la ciselure à main levée, est elle aussi particulièrement précise, grâce à de nombreux dessins, croquis et illustrations, auxquels s'ajoutent de très belles photographies dans le cahier central). S'il faut 150 gestes de percussion pour certains types de décors traditionnels, le poinçon réduit le nombre de gestes techniques à 20 . L'importante parcellisation du travail et l'interchangeabilité des agents sont un atout face aux sollicitations du marché à la recherche de rendements et de coûts de production avantageux, mais elles créent, en contrepartie, une distanciation affective des dinandiers par rapport à leur métier.

Baptiste Buob détaille les appellations terminologiques qu'il utilise pour décrire des actions techniques et les postures du corps qui leur sont associées. Les classifications proposées par Leroi-Gourhan sont croisées avec celles d'autres auteurs. Il en arrive à la conclusion suivante: «De fait, la spécialisation des ateliers fassis dans la confection de quelques objets, en l'occurrence les plateaux, a engendré une standardisation posturale, laquelle restreint le rôle des membres inférieurs du corps au soutien de pièces par la cuisse » (p. 251).

Dans la quatrième partie, Baptiste Buob interroge les travailleurs, la façon dont ils se représentent leur métier et comment ils subissent leur statut de dominés.

1. On sait ce que les productions en « deux semaines passées sur place» offrent à l'ethnographie...

2. Cour social de l'économie caravanière, commerce, dépôt, hôtel, espace pour les animaux et maison de prostitution. 
La transmission n'est pas formalisée, elle est masquée car mêlée à l'exercice de production. L'auteur aborde la présence des enfants et l'apprentissage sur le tas. Le maître prolonge cet apprentissage qui lui offre une main-d'œuvre presque gratuite. L'apprenti représente son maître à l'extérieur, c'est l'apprentissage d'un "savoir-être" avant celui d'un savoir-faire. La relation de soumission est très forte. La progression dans la hiérarchie pour l'apprenti se lit aussi dans l'espace: "Les relations de soumission prennent donc appui, de manière explicite, sur les modes d'occupation de l'espace du corps et des apprentis» (p. 294).

Aujourd'hui, on ne parle plus d'apprentis, mais d'enfants dont les ateliers ont un besoin important. Embauchés entre 7 et 14 ans, ils sont indispensables pour le portage des marchandises dans les ruelles de la Médina, va-et-vient souligné par l'auteur et fort bien illustré dans les films Ovales et Tifor. Si, autrefois, les enfants travaillaient pour apprendre un métier, ce n'est plus toujours le cas, car beaucoup le font désormais pour les besoins de leur famille. C'est ainsi tout un savoir-faire qui cesse de se transmettre car les jeunes privilégient des ateliers où les activités sont plus lucratives, comme celle du poinçonnage. En refusant de transmettre leur savoir, les dinandiers eux-mêmes manifestent leur désaccord face à ce dévoiement du savoir-faire. En conséquence de quoi, l'apprentissage est en faillite et les savoirs simplifiés. Pourquoi un tel renoncement à l'égard du métier, s'interroge l'auteur? La parole est donnée aux dinandiers.

La rétribution se fait soit à la pièce soit par un salaire hebdomadaire ou journalier (60 à 200 dirhams par semaine). Le véritable savoir-faire met les ouvriers spécialisés à l'abri de l'aliénation du travail stable et routinier. Celui trop aisément acquis (dresseurs) entraîne une forte concurrence et restreint l'esprit de corps : «La mauvaise situation de la dinanderie est couramment attribuée aux grands patrons adeptes d'un capitalisme douillet» (p. 319). Le travail probe est entravé, conclut Baptiste Buob, aussi les perspectives des dinandiers sontelles limitées. Pour la plupart, le refus du profit domine : "mieux vaut exercer une activité sans grande perspective d'avenir plutôt que de chercher à s'enrichir au détriment d'autrui " (p. 340). C'est ce que l'auteur nomme l' "idéologie de la probité " (p. 341). Comme précédemment évoqué, la « tradition » du thé au Maroc est récente, or la dinanderie s'est très vite emparée de la fabrication des ustensiles en mécanisant leur fabrication: "Qu'il s'agisse des objets de luxe ou des ustensiles du thé, la situation actuelle de la dinanderie est indissociable de l'idéologie orientaliste du protectorat et des desseins expansionnistes européens (impérialisme et capitalisme marchand) " (p. 349).

L'ouvrage s'achève par une annexe sur l'enquête d'anthropologie filmique. Baptiste Buob insiste sur le fait que le processus de réalisation et de visionnage des images fait partie intégrante de sa recherche. Les grands principes en sont les suivants: une observation filmique avouée, une absence d'intervention orale, une attitude non directive, la description suivie de processus techniques et, enfin, la volonté de rendre l'activité filmée intelligible pour le spectateur. La caméra a facilité l'insertion de l'auteur, cette observation filmique avait davantage de sens pour les acteurs que l'observation classique: "Cette profilmie [...] pourrait être l'expression de l'existence d'une conception de l'artisanat fondée sur la pièce unique dans un contexte pourtant dominé par le travail sériel et parcellisé " (p. 368). Le film saisit ce que l'observation directe ne permet pas toujours de déceler.

Un petit regret cependant, l'analyse du métier désincarne les acteurs que l'on aurait aussi aimé voir en dehors de leur travail, dans la vie quotidienne. Cela n'entame pas la qualité de l'ethnographie minutieuse et l'apport essentiel de l'ouvrage à l'anthropologie des techniques et à l'anthropologie visuelle.

Marie-Luce Gélard 


\section{E U R O P E}

\section{Made in Sheffield. An Ethnography of Industrial Work and Politics New York-Oxford, Berghahn Books, 2009, 2 I 2 p., ill., bibl., index (« Dislocations »).}

\section{C}

E LIVRE s'inscrit dans la collection "Dislocations" conçue comme un forum d'expression pour "des réactions politiquement engagées, ethnographiquement informées et théoriquement percutantes " aux conséquences humaines, sociales et économiques de la "globalisation néolibérale et du recul de l'État providence». L'auteur définit en effet Made in Sheffield "d'abord et avant tout comme une ethnographie de la conscience ouvrière » (p. 2) s'appuyant sur deux études de terrain dans ce qu'il reste de l'univers industriel de l'ancienne capitale de l'acier et de la coutellerie. À l'instar d'autres anthropologues ayant enquêté en Angleterre ${ }^{1}$, Massimiliano Mollona a pris au pied de la lettre l'expression "ethnographie participante» : déterminé à pénétrer un monde réticent à l'intrusion d'universitaires, il a fait un détour par la pêche à la ligne, loisir " ouvrier " par excellence, pour s'en approcher et réussir à être recruté comme apprenti dans une usine d'outillage où il a ensuite travaillé au rythme de huit heures par jour, deux jours par semaine pendant dix-huit mois. Il a poursuivi avec un emploi à temps complet dans une petite aciérie pendant un an.

Il a renforcé cette immersion au quotidien par un partage des loisirs des ouvriers de la première entreprise (sorties au pub, parties de billard, pêche à la ligne...) et par une installation, pendant les derniers mois de l'enquête, dans une pension plus ou moins clandestine du quartier hébergeant travailleurs immigrés, chômeurs, trafiquants de drogue et souteneurs. En outre, mettant ses convictions personnelles et son expérience de militant au service de ses informateurs, il a participé activement à la vie politique de la seconde entreprise, animant des débats en interne, mais aussi entre les ouvriers et les chercheurs et avec les médias, interpellant les représentants des industriels, manifestant avec les ouvriers et faisant du lobbying à Westminster et dans les ministères. Il a également réalisé un film ${ }^{2}$ sur le travail, en associant les ouvriers au montage.

1. Nigel Rapport a travaillé comme garçon de ferme chez des éleveurs du Yorkshire (cf. Diverse World-Views in an English Village, Edinburgh, Edinburgh University Press, 1993) puis comme brancardier dans un hôpital écossais (cf. «Je suis peut-être un salaud d'Anglais, mais je suis pas un feignant !", Ethnologie française, 2007, 37 [2] : 255-264). Jenny Hockey a occupé un emploi d'aide-soignante dans une maison de retraite (cf. "Interviews as Ethnography? Disembodied Social Interaction in Britain ", in Nigel Rapport, ed., British Subjects. An Anthropology of Britain, Oxford, Berg, 2002 : 209-222).

2. Intitulé Steel Lives, "Vies de l'acier" (jeu de mots avec Still Life, "Nature morte ») et projeté à Sheffield en 2000, lors du Festival international du documentaire. 
Le fruit de cet engagement multiforme est un ouvrage " inspiré par l'ethnographie du capitalisme victorien de Marx» (p. 2), s'appuyant sur les acquis de l'école de Chicago et de l'école de Manchester. «Combinant anthropologie et analyse des rapports de classe» (p. 1), l'auteur bat en brèche ce qu'il considère comme le «mythe de la disparition de la classe ouvrière" entretenu par des historiens "révisionnistes", pp. 167 sqq.). Par la richesse et la rigueur de la restitution, le livre est une source documentaire précieuse sur les transformations du travail et de la main-d'œuvre consécutives à la désindustrialisation et à la dérégulation advenues en Angleterre dès la fin des années 1970. En même temps qu'il considère le phénomène connexe de perte de pouvoir économique et politique des industriels de l'acier au profit d' "une nouvelle élite de développeurs planétaires, spéculateurs urbains et bureaucrates gouvernementaux» (p. 86), Massimiliano Mollona propose une analyse du syndicalisme pendant cette même période. Grâce à cette mise en perspective temporelle et à des changements d'échelle, allant de la base - le shop floor, l'atelier - au Parlement, l'auteur prend en compte aussi bien les idiosyncrasies individuelles ${ }^{3}$ que les relations sociales, le corps au travail que le marché de l'acier, pour comprendre les rapports des ouvriers aux machines et à la matière, mais aussi entre eux et avec leurs employeurs. Sont également examinées les relations des ouvriers à la famille, au voisinage de même qu'à la société dans son ensemble.

Dans la première entreprise, assimilée par Massimiliano Mollona à « une échoppe de sueur victorienne» (p. 19) caractérisée par la précarité, la dérégulation fiscale et une machinerie obsolète, et où la direction joue de tous les mécanismes juridiques disponibles pour produire des profits tout en faisant reposer la responsabilité de la survie de l'affaire sur les ouvriers, l'auteur fait ressortir des différences radicales de perception de la "valeur travail", selon qu'on considère l'espace "chaud" des ouvriers fondant ou forgeant l'acier, comme leurs pères et grands-pères avant eux, soucieux de reproduire une culture professionnelle de valorisation du geste et de la machine, et de résistance à l'autorité, ou celui, "froid", des affûteurs et polisseurs appartenant à une nouvelle génération dépolitisée, beaucoup plus scolarisée, distanciée du travail en termes symboliques, techniques et sociaux, et en quête de rendement et de bonus. Au jour le jour, les deux groupes, contraints de coopérer dans l'enceinte de l'usine, maintiennent un compromis, notamment en ce qui concerne la cadence, au bénéfice de l'employeur.

Les premiers sont des hommes d'âge mûr qui, sur le shop floor, contrôlent le recrutement et la formation des apprentis tout en gardant une certaine main mise sur le marché; à l'extérieur, ils participent d'une culture ouvrière par un mode de vie et des valeurs qui, par exemple, subordonnent les juniors et les femmes. Les seconds, quant à eux, séparent lieu de travail et quartier de résidence, et visent un certain embourgeoisement; mieux rémunérés, mais dépendants de leurs seuls salaires, ils sont en même temps plus vulnérables. Aussi Massimiliano Mollona les considère-t-il comme des "prolétaires " aliénés par le travail tandis qu'il définit les seniors comme des artisans, en français dans le texte (pp. 80-81). On comprend la fonction rhétorique d'un emprunt qui reste cependant problématique: dans son contexte d'origine (l'économie française), le vocable artisan désigne bien des travailleurs manuels spécialisés fiers de leur savoir-faire mais qui sont leur propre employeur.

Socialement, le terme est encore plus inadéquat quand on considère d'autres

3. Voir les deux portraits saisissants à la fois de recul et d'empathie qu'il brosse: d'un militant "stakhanoviste et collectiviste" dont la fierté des origines ouvrières le dispute au rêve finalement avorté d'accéder à un emploi non manuel; puis d'un travailleur italien tiraillé entre son identification aux "gars de l'atelier", ses aspirations à une vie de petit-bourgeois et son ambivalence eu égard à son milieu d'origine. 
critères contribuant à l'identité du groupe. Ces ouvriers parviennent en effet à un relatif équilibre financier grâce à des ressources parallèles : outre le négoce au marché noir de "déchets " d'acier prélevés dans l'usine, ils honorent des commandes passées par des sous-traitants en utilisant l'outillage de l'atelier, avec l'accord tacite de la direction. Eux et leurs proches sont par ailleurs impliqués à titres divers dans l'économie illicite (travail des mineurs, prostitution, recel, drogue) de la zone péri-urbaine où ils travaillent et habitent ; zone au sol contaminé par trois siècles d'activité industrielle et que les pouvoirs publics et les promoteurs n'ont pas encore " réaménagée " au moment de l'enquête. $\mathrm{La}$ complémentarité des diverses activités dans ce quartier de non-droit permet à différentes populations (Anglais "de souche", et Yéménites et Pakistanais attirés cinquante ans auparavant par l'industrie de l'acier) de cohabiter voire de collaborer face à d'éventuels contrôles des services sanitaires, fiscaux ou policiers. Ainsi est préservée une indépendance relative et fragile qui, pour un temps, neutralise les efforts bien intentionnés des pouvoirs publics pour "reclasser» ces résidents marginaux, en particulier dans le secteur tertiaire. Perçue comme un déni de l'expertise et de la mémoire ouvrières, la perspective de ces emplois « réservés » est d'autant plus humiliante qu'elle va de pair avec une démarche de réhabilitation urbaine, c'est-à-dire d'effacement, d'un espace à forte identité.

Par les questionnements théoriques et épistémologiques qu'il examine, Made in Sheffield intéressera, outre les anthropologues, les économistes, politologues et historiens. Sans dogmatisme, l'auteur porte un regard à la fois humain et distancié sur le quotidien précaire - parfois drôle, souvent tragique - d'une population oubliée. En même temps, à partir de ces matériaux de première main, il propose une réflexion originale sur un moment essentiel de l'histoire industrielle et sociale européenne. 